\title{
Balkanologie
}

Balkanologie Revue d'études pluridisciplinaires

Vol. I, n 1 | 1997

Volume I Numéro 1

\section{Citoyenneté dans un État pluri-national}

Le cas de l'ex-Yougoslavie

\section{Michel Drouet}

\section{OpenEdition}

Journals

Édition électronique

URL : https://journals.openedition.org/balkanologie/204

DOI : 10.4000/balkanologie.204

ISSN : 1965-0582

Éditeur

Association française d'études sur les Balkans (Afebalk)

\section{Édition imprimée}

Date de publication : 1 juillet 1997

ISSN : 1279-7952

\section{Référence électronique}

Michel Drouet, « Citoyenneté dans un État pluri-national », Balkanologie [En ligne], Vol. I, nº 1 | 1997, mis en ligne le 02 juin 2008, consulté le 28 juin 2022. URL : http://journals.openedition.org/balkanologie/ 204 ; DOI : https://doi.org/10.4000/balkanologie.204

Ce document a été généré automatiquement le 17 décembre 2020.

(c) Tous droits réservés 


\section{Citoyenneté dans un État pluri- national}

Le cas de l'ex-Yougoslavie

Michel Drouet

1 L'ex-Yougoslavie fédérale a pu longtemps apparaître comme un modèle de traitement des questions nationales ${ }^{1}$, questions devenues essentielles dans cette partie de l'Europe à partir du XIX ${ }^{\text {ème }}$ siècle, lorsque le déclin puis la disparition des Empires ottoman et austro-hongrois ont donné naissance à des États composés de plusieurs peuples. Ce modèle a permis en 1945 la reconstruction de la Yougoslavie disparue en avril 1941 avec d'une part son occupation par les troupes allemandes et leurs alliés italiens, hongrois et bulgares, et d'autre part la création, sous l'égide allemande, d'un "État indépendant croate". Il assura pendant de longues années la coexistence pacifique entre les diverses nationalités, en particulier entre Serbes et Croates dont les relations ont été décisives pour le devenir de la Yougoslavie dès sa constitution.

\section{La question nationale dans l'ex-Yougoslavie}

Les relations entre ces deux principaux peuples avaient été marquées jusqu'alors par deux sources d'antagonisme.

Dès sa création, en 1918, existent deux conceptions du "yougoslavisme"2. L'une, que l'on retrouve principalement auprès des élites croates, prônait un État décentralisé, fédéral, respectueux des particularismes nationaux et notamment des institutions politiques spécifiques que les Croates avaient obtenues dans le cadre de l'Empire austro-hongrois: duc-gouverneur (Ban), assemblée (Sabor) et milice locale (Domobranstvo). L'autre, portée par la royauté d'origine serbe, visait à créer un ÉtatNation unitaire et centralisé sur le modèle français, en particulier à partir de 1929 lorsque le Royaume des Serbes, Croates et Slovènes prend le nom de Yougoslavie et que le régime constitutionnel est aboli au profit d'une dictature royale. Cette évolution laissera un profond sentiment de frustration aux élites croates, malgré une grande 
autonomie politique accordée en 1939 à la Croatie, avec le rétablissement de ses institutions de l'époque austro-hongroise ${ }^{3}$.

4 L'autre source d'antagonisme découle de la politique raciste menée par "l'État indépendant croate" sur ses territoires comprenant approximativement l'actuelle Croatie sans la côte dalmate, ainsi que l'actuelle Bosnie-Herzégovine. Les victimes de cette politique mise en oeuvre dès le printemps 1941 seront certes les juifs et les Tsiganes mais aussi, et surtout par le nombre des victimes, les Serbes, conformément au "programme" énoncé alors par Mile Budak, ministre oustachi des Cultes et de l'Éducation: "Nous tuerons une partie des Serbes, une autre partie nous l'expulserons et le reste nous les convertirons à la religion catholique et les transformerons en Croates $»^{4}$.

5 La question nationale en Europe centrale et orientale a conduit à maintes reprises, au $\mathrm{XIX}^{\mathrm{ème}}$ mais surtout au XX ${ }^{\text {ème }}$ siècle avec l'effondrement des Empires, à des transferts et / ou expulsions de populations au nom de l'homogénéisation souhaitée / forcée d'un territoire: populations turques et islamisées, minorités allemandes, pour ne retenir que les plus importantes... ${ }^{5}$ Ces pratiques ont également concerné des populations de l'actuelle Yougoslavie, lors du déclin de l'Empire ottoman, notamment entre Serbes et Albanais. Mais en recourant aux massacres systématiques, le régime oustachi innovait tragiquement, d'autant plus qu'il les commettait à l'égard d'une population avec laquelle les Croates n'avaient pas "d'antagonisme séculaire" ni même de récents conflits armés directs.

6 Dans un tel contexte, l'organisation fédérale mise en place à partir de 1945 réussira non seulement à apaiser les tensions entre Serbes et Croates mais aussi à assurer aux autres peuples des droits qu'ils ne connaissaient pas dans la Yougoslavie royale. Pour une part, cet apaisement était lié au monolithisme politique propre au régime communiste et à ses capacités répressives ${ }^{6}$. Mais il résultait également de la légitimité du Parti communiste acquise au cours de la résistance à l'occupant, résistance qui, explicitement, reposait sur l'ensemble des peuples de la Yougoslavie et le dépassement de leurs antagonismes ${ }^{7}$; le slogan officiel « fraternité et unité » sera pendant longtemps d'autant mieux reçu qu'il s'accompagnait de cette organisation fédérale et d'un processus intense de modernisation économique et sociale.

7 Néanmoins, les premières élections pluralistes depuis la prise du pouvoir par le Parti communiste, tenues séparément dans les différentes Républiques de la Fédération au cours de 1990, se traduiront par l'arrivée au pouvoir d'hommes politiques qui pour la plupart d'entre eux avaient mis en avant des discours nationalistes, certains prônant même l'indépendance. Comme on le sait, Slovénie et Croatie la proclameront le 25 juin 1991, ce qui sera entériné internationalement par l'Allemagne puis par la CEE au début de 1992; suivront la reconnaissance de la Bosnie-Herzégovine (avril 1992) et de la Macédoine (avril 1993). La dislocation de la Yougoslavie débouchera même sur un conflit armé en Croatie et en Bosnie-Herzégovine.

8 L'échec du modèle fédéral semble donc patent et la question nationale demeure centrale d'autant que ces nouveaux États comportent tous des minorités importantes, excepté la Slovénie presque homogène ethniquement. Cerner les facteurs de cet échec semble d'autant plus important qu'il conditionne les choix politiques et institutionnels des nouveaux États. Il met en cause, sur le plan politique, les conditions d'exercice de la citoyenneté. Celles-ci dépendent de leur cadre institutionnel, des pratiques politiques 
dominées par le monolithisme du Parti communiste jusqu'à la fin des années 1980 ainsi que des attitudes de la population dans ce contexte.

\section{La citoyenneté dans l'État fédéral}

Sur le plan politique, la citoyenneté, entendue au sens large d'appartenance à un Étatnation, était duale : fédérale et relevant des différentes Républiques, au nombre de six.

La définition des Républiques était avant tout territoriale mais également ethnique puisqu'à chaque République correspondait de fait un "peuple constitutif" de la Yougoslavie (narod ou "nation"), d'où l'appellation même de la République (SlovènesSlovénie etc.). Chaque République apparaissait ainsi comme celle d'une nation spécifique, majoritaire, et celle des membres des autres peuples ${ }^{8}$. Aux trois peuples fondateurs (Serbes, Croates et Slovènes) de 1918, avaient été joints par la Constitution de 1946 les Monténégrins et les Macédoniens, ces derniers se voyant reconnus pour la première fois comme un peuple spécifique, d'où l'existence de cinq Républiques. En l'absence de "nation" majoritaire, seule la Bosnie-Herzégovine échappait à ce schéma ${ }^{9}$; elle sera ainsi le territoire de trois "nations" ou "peuples constitutifs" : les Serbes, les Croates et les Musulmans, ces derniers ne se voyant cependant reconnaître le statut de “nation-peuple" qu'à la fin des années 1960.

11 À ces différentes "nations" s'ajoutaient une vingtaine de "nationalités" (narodnost), minorités définies soit comme des peuples ayant leur "nation-mère" dans un État voisin, en premier lieu les Albanais et les Hongrois, soit comme des minorités "transnationales" tels que les juifs ou les Tsiganes ${ }^{10}$.

La distinction entre "nation" et "nationalité" établissait donc deux catégories de citoyens sur des bases ethnico-culturelles, avec pour la "nation" la possibilité de prétendre être celle d'un État-nation / République défini alors ethniquement. C'est précisément au nom de cette différence que sera rejetée la revendication, énoncée dès 1968 et plus encore en 1981, des Albanais de voir le Kosovo reconnu non plus seulement comme une Province autonome de la République de Serbie mais comme une République à part entière ${ }^{11}$ : ils n'étaient en effet qu'une "nationalité" du fait de l'existence de l'Albanie .

Précisons cependant que la Constitution fédérale de $1974^{12}$ relativise la portée de cette distinction en posant comme principe l'égalité en droits entre "nations" et "nationalités", en affirmant la supériorité de la citoyenneté yougoslave sur celle des différentes Républiques d'où égalité des droits et devoirs entre le citoyen d'une République vivant dans une autre République et ceux de cette dernière. Était également reconnue à chaque citoyen la liberté d'appartenance à une nation ou une nationalité (avec celle d'usage de sa langue et de son alphabet, même dans ses rapports avec les organismes étatiques, et celle d'enseignement dans sa langue), liberté qui comprenait le droit de refuser d'opter pour une appartenance.

14 La définition politique était de plus relativisée par sa définition sociale, d'inspiration marxiste ; ainsi le coeur de la citoyenneté paraît être le "travailleur associé", figure emblématique de l'autogestion, l'État fédéral étant défini comme une "communauté étatique de nations librement unies (...) fondée sur le pouvoir et l'autogestion de la classe ouvrière et de tous les travailleurs» (art. 1). Pas moins de 142 articles de la Constitution, sur un total, il est vrai, de 406 articles..., étaient ainsi consacrés à la 
codification de l'autogestion et précédaient les articles consacrés à l'organisation politique.

15 Au-delà des catégories juridico-politiques, il faut souligner la prégnance de la référence ethnico-culturelle de la citoyenneté, notamment sa référence confessionnelle, et le flou de la notion de "nation" souvent entendue comme celle de peuple sans lien univoque avec un État / République aux frontières précisément définies. Ainsi en allait-il des Serbes disséminés (le quart d'entre eux au recensement de 1991) pour des raisons historiques sur les territoires de plusieurs autres Républiques; les Serbes de Croatie, par exemple, se considéraient assurément Serbes et / ou citoyens yougoslaves avant d'être citoyens croates. De même, les Albanais du Kosovo, citoyens yougoslaves et de la République de Serbie, pouvaient se revendiquer Albanais (mais cette citoyenneté n'était reconnue que comme celle d'une minorité ou "nationalité"), quant à s'identifier à la citoyenneté serbe...

16 Encore plus confuse paraissait être la "nation" Musulmane concernant une des communautés de Bosnie-Herzégovine tout autant slave et parlant la même langue que les deux autres communautés de cette République, les Serbes et les Croates ${ }^{13}$. Le principe identitaire mis alors en avant est l'islam, même si l'argumentaire officiel, lors du recensement de 1971, ne retenait que l'idée de groupe ethnique institutionnalisé en "nation"14. Mais les membres de cette "nation" peuvent être laïcs, voire non croyants, alors que des musulmans, conformément à leur détermination antérieure à la création de l'ethnie puis de la "nation" musulmane, peuvent continuer à se déclarer Serbes ou Croates. En outre, cette "nation" ne regroupe pas l'ensemble des musulmans de Yougoslavie puisque, notamment, les Albanais, de confession musulmane pour la quasitotalité, étaient considérés comme des citoyens serbes membres de la "nationalité" albanaise. Nous reviendrons ultérieurement sur les implications de la création, tardive, de cette "nation".

\section{De la Fédération à la Confédération}

17 La dualité de la citoyenneté va cependant évoluer en fonction du glissement progressif, à partir du milieu des années 1960, d'une organisation politique fédérale à une organisation confédérale avec la montée en puissance des Républiques au détriment des organes fédéraux. Le déclin de la citoyenneté yougoslave se renforcera après la mort de Tito (1980), lorsque se développeront les revendications nationales, entendues au sens d'État-nation défini par rapport à une "nation", donc à base ethnico-culturelle, ce qui conduira à la dislocation de la Yougoslavie en 1991.

Cette évolution est amorcée avec la réforme économique de 1965-1966 qui renonce à toute planification et veut promouvoir l'économie de marché, dans le contexte de la propriété collective des moyens de production et de l'autogestion. En résultera l'affaiblissement du pouvoir économique de l'État central et le développement dans un premier temps des mécanismes de marché mais aussi, et de façon croissante, du régionalisme économique dans le cadre des Républiques comme des communes; ainsi l'un des thèmes constants tout au long des années 1970 et 1980 sera la constitution d'un marché unique yougoslave pour faire pièce au protectionnisme des autorités locales. Sur le plan institutionnel, le tournant vers une Confédération s'opère avec les amendements constitutionnels de 1971 et la Constitution de 1974, en vigueur jusqu'à la 
dislocation de la Yougoslavie fédérale. Le caractère hybride des dispositions retenues par ces textes permet de considérer qu'ils instaurent un fédéralisme dénaturé ${ }^{15}$.

Postulant dans son préambule le droit de chaque peuple à l'autodétermination, y compris le droit à la sécession, la Constitution précise dans son article $1^{\text {er }}$ que la Yougoslavie est un État fédéral, «communauté étatique de nations librement unies et de leurs Républiques...ainsi que des Provinces... », la République étant " un État fondé sur la souveraineté de la nation...» (art. 3). Cette souveraineté, de nature confédérale, est cependant limitée puisque « les frontières de la République socialiste fédérative de Yougoslavie ne peuvent être modifiées sans l'accord de toutes les Républiques et Provinces... » (art. 5) ${ }^{16}$.

Les organes fédéraux (exécutif et législatif) paraissent, néanmoins, n'être que l'émanation des Républiques et Provinces ${ }^{17}$.

21 Ainsi, la Présidence fédérale est un collège composé du président du Parti communiste yougoslave et, sur une base paritaire, d'un représentant de chaque République et Province. Ces représentants ne sont cependant pas élus au suffrage universel direct, ni même par l'Assemblée fédérale, mais par l'Assemblée de chaque République / Province qui, seule, peut les révoquer.

22 Le président de ce collège est lui-même nommé par ses pairs selon le principe de la rotation annuelle; cette disposition n'entrera réellement en vigueur qu'en 1980, Tito ayant été nommé en 1974 Président à vie. Le caractère collégial de la Présidence, renforcé par la rotation annuelle, conduit cependant à sa paralysie dès lors que le consensus entre ses membres, en fait entre les Républiques / Provinces, est rompu. Ce consensus repose en réalité, et en dernier ressort, sur l'appartenance de ses membres au Parti unique, communiste, les divergences étant soumises à ses règles de discipline interne, en particulier à l'arbitrage de Tito. Mais ce Parti se décomposera à la fin des années 1980 et les élections pluralistes de 1990 vont permettre l'arrivée au pouvoir dans les Républiques de partis spécifiques à chacune d'entre elles et ... nationalistes. Dès lors, comme on pourra le constater en 1991, la Présidence sera incapable de remplir ses attributions qui, outre celles honorifiques, concernaient l'harmonisation des intérêts des Républiques / Provinces et le commandement suprême de l'armée ${ }^{18}$.

Les chambres fédérales correspondent elles aussi au principe de la représentation paritaire des Républiques et des Provinces. Les membres du Conseil fédéral, sorte de Chambre des représentants, sont élus au suffrage universel direct mais par République (30 représentants chacune) ou par Province (20), alors que ceux du Conseil des Républiques et des Provinces sont nommés par chaque République (12 membres) et chaque Province ( 8 membres). Seule entorse au principe paritaire, la représentation minorisée des Provinces par rapport aux Républiques est elle-même source de conflits potentiels puisque ces Provinces acquièrent, ainsi et avec leur représentation dans le collège présidentiel, un statut similaire à celui des Républiques bien que faisant partie de la République de Serbie...

24 Il est habituel dans une Fédération qu'une des deux chambres représente ses différentes composantes territoriales sur une base paritaire et que l'autre chambre, visant à affirmer l'identité fédérale, représente l'ensemble de la population sur une base principalement démographique. Tel ne pouvait être le cas du Conseil fédéral dont la composition découlait des Républiques / Provinces, chacune étant considérée comme 
ayant le même poids, alors que leur importance démographique pouvait aller de 1 à 9,5 (Serbie sans ses Provinces comparée au Monténégro).

L'application du principe de parité au Conseil fédéral ne laissait ainsi guère d'espace à l'expression de la citoyenneté fédérale. L'importance de cette absence n'apparaîtra pleinement qu'en 1990, avec les premières élections pluralistes d'après-guerre. Jusqu'alors, en effet, les représentants des différentes Républiques / Provinces étaient élus sur une liste de candidats sélectionnés par "l'Alliance socialiste", organisation de masse relais du Parti communiste, ce qui permettait d'assurer une identité autre que celle propre à chaque République / Province. Dès lors que le monolithisme politique disparait en 1990, l'identité propre à chaque République / Province l'emporte sur les affinités idéologiques trans-républiques; en témoignent la quasi-inexistence de partis trans-républiques et l'échec du parti affichant son identité yougoslave, ainsi que le rejet de tout projet tendant à supprimer le principe de parité pour le Conseil fédéral en vue de faire prévaloir des critères démographiques ${ }^{19}$.

Globalement, l'application du principe de représentation paritaire des Républiques / Provinces à l'exécutif et au législatif fédéral, à l'exclusion de tout élément de pondération hormis celle entre Républiques et Provinces, ne fait de ces organes que l'émanation des composantes de la Fédération, les privant de légitimité politique propre et empêchant l'expression politique de la citoyenneté yougoslave. On peut alors affirmer ${ }^{20}$ que la Constitution de 1974 consacre le passage d'une fédération de "nations" (au sens ethnico-culturel) à une confédération de territoires; encore faut-il préciser que ceux-ci, les Républiques / Provinces, renvoient chacune, hormis la BosnieHerzégovine, à une "nation" majoritaire avec des minorités.

L'orientation confédérale est au demeurant renforcée par les pratiques décisionnelles au sein de ces organes, où va prévaloir, au-delà des textes constitutionnels, la règle du consensus voire de l'unanimitée ${ }^{11}$. Cette pratique concernera la Présidence, dès la disparition de Tito, et tendra à se généraliser à l'ensemble des organes fédéraux d'où un droit de veto de fait pour les différentes composantes de la Fédération en cas de divergences prononcées.

En découlera notamment la difficulté à mettre en oeuvre une politique macroéconomique d'ajustement face à la crise de la dette extérieure des années $1980^{22}$. La définition des mesures d'ajustement, la répartition des inévitables mesures d'austérité, seront le terrain de tensions entre Républiques. Leur mise en oeuvre, partielle, reflétant la faiblesse de la capacité régulatrice du pouvoir central, conduira à la stagnation économique et à une inflation croissante, sources elles-mêmes de tensions socio-économiques. Les discours nationalistes, dans un premier temps à connotation économique, seront ainsi orientés vers la défense des intérêts propres à chaque République et iront à la rencontre du désenchantement de la population. Face à l'effondrement d'un des mythes de la Yougoslavie d'après 1945, l'autogestion et sa capacité à assurer la transformation économique et sociale, le repli identitaire sur la République débouchera rapidement sur le repli identitaire ethnique, la République étant assimilée à sa "nation" majoritaire .

\section{L'instrumentalisation de la question nationale}

Le monolithisme du parti communiste fut de fait le garant de l'unité nationale jusqu'au cours des années 1980. Le coeur du pouvoir résidait en effet dans ses organes dirigeants 
dont le principe de fonctionnement reposait sur l'unité d'idées d'où la quasiimpossibilité de conflits ouverts; cette unité était en outre garantie par Tito. Ceci n'excluait pas l'instrumentalisation de la question nationale par le Parti communiste dont les conséquences serviront de ressort au renouveau des nationalismes dans les années 1980.

Ainsi en est-il allé de l'affaiblissement délibéré de la République de Serbie, selon le principe, non affiché il va de soi, qu'une Yougoslavie forte supposait une Serbie affaiblie puisque l'échec de la Yougoslavie royale était attribué à "l'hégémonie" serbe ${ }^{23}$.

31 L'organisation fédérale de 1945-1946 passait donc par un certain rééquilibrage. La Serbie fut diminuée avec la création de la République de Macédoine, qui faisait partie de la Serbie depuis les guerres balkaniques de 1913. Le Monténégro, dont le Parlement avait voté le rattachement à la Serbie à la fin de la Première Guerre mondiale, redevint un État. Enfin, au sein de la Serbie ainsi réduite, furent instaurées deux Provinces autonomes au nom de la présence de minorités, importante au point d'être majoritaire au Kosovo, multiples mais au total minoritaires en Voïvodine ; ce principe ne sera pas appliqué à la Croatie, qui avait une minorité italienne, hongroise et surtout serbe, ni à la Macédoine en faveur de sa minorité albanaise.

Comme nous l'avons vu, la présence des représentants de ces deux Provinces au sein des organes fédéraux leur conférait un statut proche de celui d'une République, bien que faisant toujours partie de la République de Serbie ; avec la pratique du consensus / unanimisme, cette position leur donnait un droit de veto, y compris à l'égard de leur propre République... Cette situation, ambiguë si ce n'est incohérente, sera une des sources majeures du développement du nationalisme serbe dans les années 1980, dans le contexte de la revendication de la minorité albanaise de voir le Kosovo érigé en République.

Nous avons déjà souligné le caractère confus de la "nation" musulmane. La transformation d'un groupe confessionnel en ethnie puis en "nation" dotée de droits spécifiques est exemplaire du fait que les ethnies pas plus que les nations ne sont naturelles mais qu'elles sont le produit d'une situation politique ${ }^{24}$. D'une caractéristique confessionnelle, le régime fera une ethnie pour tous ceux qui, relevant de cette confession, n'appartenaient pas à une autre ethnie; mais, ce faisant, il permettait également de développer cette identité ethnique à ceux, qui tout en étant originaires de ce groupe confessionnel, ne voulaient pas se définir par rapport à cette seule référence, par exemple aux communistes d'origine musulmane et plus généralement aux musulmans "laïcs". En dotant ultérieurement cette ethnie d'un statut de "nation" et donc de "peuple constitutif", le régime avait pour but d'empêcher la confrontation entre Croates et Serbes en Bosnie-Herzégovine et plus généralement de la réduire dans l'ensemble de la Fédération; par ailleurs, il renforçait son aura sur le plan international, principalement auprès des pays musulmans au régime laïc au sein du mouvement des pays non alignés dont il fut un des promoteurs ${ }^{25}$.

Les autorités musulmanes religieuses mettront à profit ce contexte pour développer leurs activités en Bosnie-Herzégovine (presse, écoles religieuses, construction de mosquées, ...) et leurs relations internationales avec les divers organismes islamiques. Mais ce renouveau religieux important servira de cadre légal à l'essor du nationalisme musulman en Bosnie-Herzégovine, allant ainsi à la rencontre d'une partie de l'intelligentsia musulmane locale. Celle-ci, initialement préoccupée par la revalorisation du passé culturel musulman et par la construction de mythes fondateurs 
de la "nation" musulmane, débouchera sur les positions du nationalisme dit laïc voire progressiste, que l'on retrouvera également parmi des dirigeants locaux du Parti communiste.

Cette situation apparaîtra au grand jour à la fin des années 1970 et au début des années 1980. Le régime y répondra par ses habituelles méthodes répressives, pour l'essentiel "douces" (campagne de propagande, changement de dirigeants, etc.). Elles culmineront en 1983 avec des peines de prison " pour activités contre-révolutionnaires d'inspiration nationaliste musulmane » d'un groupe réuni autour d'une Déclaration islamique dont l'auteur, A. Izetbegović, sera l'un des fondateurs et le dirigeant du parti musulman (SDA) pour lequel les Musulmans voteront très largement en 1990 ; il deviendra alors Président de la Bosnie-Herzégovine ${ }^{26}$.

Dernier exemple d'instrumentalisation de la question nationale, mais non le moindre puisqu'il débouchera sur les changements institutionnels de 1971 et 1974: la "crise croate de 1971" ${ }^{27}$. Cette crise était le produit de la convergence de revendications nationales de nature culturelle et économique. À partir de 1967, les revendications culturelles concernant les minorités croates dans les autres Républiques seront propagées par une institution culturelle, Matica Hrvatska, qui reprendra la thèse de la spécificité de la langue croate, qu'il conviendrait de distinguer du serbe, contrairement à l'accord de 1850 ayant fondé le serbo-croate. Cette affirmation identitaire sera prolongée par des discours d'historiens relativisant la période oustachie, notamment l'importance de l'écho que ce régime avait pu avoir dans le peuple croate ; l'un de ces historiens sera d'ailleurs F. Tudjman, ancien général et actuel Président de la Croatie.

37 Parallèlement, dans le contexte du développement de l'économie de marché depuis la réforme de 1965 et la décentralisation économique qui s'ensuivit, les revendications économiques régionales-républicaines vont se développer et seront prises en charge par les dirigeants communistes locaux. Elles porteront sur l'attribution des crédits à l'investissement par les banques, estimées inféodées à "Belgrade" 28 et par là défavorisant la Croatie, et plus encore, les devises dont la répartition administrative se serait faite au détriment de la Croatie ${ }^{29}$. La conjonction de ces discours, culturels et économiques, débouchera en 1971 sur un mouvement politique, initialement estudiantin mais ultérieurement appuyé par des dirigeants communistes locaux, mêlant revendications démocratiques et nationalistes; la concrétisation de celles-ci aurait conduit à la sécession de la Croatie ${ }^{30}$.

38 La réaction du régime, de Tito en premier, sera double : purges au sein du personnel politique, satisfaction partielle des revendications nationalistes, notamment sur le plan économique. Mais les purges dans la direction du Parti communiste croate seront accompagnées, selon la logique de l'équilibre national dans la répression, du changement de dirigeants dans d'autres Républiques, Slovénie, Macédoine et Serbie. Ces dirigeants, surtout dans cette dernière République, prônaient, dans le prolongement de la réforme économique "libérale" de 1965, une libéralisation de la vie politique qui pouvait conduire à la remise en cause du monolithisme du Parti communiste ; leur révocation a donc permis de faire le pendant à celle des dirigeants croates tout en évacuant la question du monopole politique du parti communiste.

39 Au-delà des purges, le régime répondra à ces tensions par les amendements constitutionnels de 1971 et la nouvelle Constitution de 1974. Accroissant l'autonomie des Républiques / Provinces conforme à l'orientation confédérale de ces changements institutionnels, le régime confortait le pouvoir des différentes bureaucraties 
régionales; il institutionnalisait le nationalisme tout en le limitant, tant que le Parti communiste demeurait "la force dirigeante" et était animé d'une idéologie relativement homogène, au plan économique et social, nourrissant ainsi la fragmentation locale du marché yougoslave précédemment évoquée.

Un mode d'exercice essentiel de ce pouvoir concernera alors son rôle dans l'attribution des postes de direction, dans les institutions politiques avec leurs organes administratifs bien entendu, mais aussi dans les organisations économiques, en particulier dans les grandes entreprises. La pratique de la répartition de ces postes au niveau fédéral selon une "clef" ( $k l j u c ̌)$ respectant sur une base paritaire l'équilibre entre les Républiques sera systématisée, y compris, bien que de façon atténuée, dans l'armée. Ainsi, les possibilités de promotion sociale liées à l'appartenance au parti communiste seront prises en charge de fait par les Républiques / Provinces, contraignant les individus à faire acte d'allégeance sur cette base régionale, et en dernier ressort ethnique, compte tenu de la force de la définition ethnico-culturelle des Républiques ; dans ces conditions, revendiquer son identité yougoslave constituait un handicap pour une carrière. Ce rôle des Républiques / Provinces sera élargi par le principe de la rotation annuelle des cadres et, dans le contexte de la stagnation des années 1980, par la baisse des autres possibilités de promotion sociale ${ }^{31}$.

41 La faiblesse du pouvoir central découlant de ces changements institutionnels sera donc amplifiée par l'absence d'appareil d'État autonome à l'échelle fédérale, en mesure de faire valoir les intérêts fédéraux; seule l'armée pourra être considérée comme un appareil fédéral. La «féodalisation des structures du Parti $»^{32}$ sera par ailleurs le terreau de discordes entre dirigeants des différentes Républiques, de plus en plus ouvertes après la mort de Tito, et conduira à la fin des années 80 à la disparition du discours communiste transnational et à la dislocation du parti communiste en 1990 ; cette même année verra la multiplication des décisions unilatérales prises par les différentes Républiques au détriment de la Fédération, annonçant sa disparition.

\section{L'exercice de la citoyenneté yougoslave}

42 L'évolution vers une Confédération et le poids croissant des Républiques / Provinces comme cadre de la vie politique effective réduisaient l'espace pour l'identité yougoslave. Compte tenu du développement du régionalisme économique depuis la réforme économique de 1965, l'approche des questions socio-économiques, dont la gravité avait brutalement augmenté au début des années 80 avec le poids de la dette extérieure, sera déterminée par les intérêts propres à chaque République, tels du moins que les concevaient les élites politiques régionales.

Mais la force de la référence ethnique dans la définition des Républiques conduira à remettre au premier plan les clivages ethniques. Ainsi, l'interprétation ethnique des divergences économiques et des conflits sociaux va tendre à l'emporter sur leur approche politique, en termes de mise en cause des élites et du système politique et économique. Pour autant, les tensions interethniques restèrent fort limitées sur le plan interindividuel jusqu'à la fin des années 80 , si l'on excepte la situation prévalant au Kosovo entre Serbes et Albanais. On assiste ainsi, au cours des années 80 , à un poids croissant des références ethniques dans la sphère politique, y compris dans le cadre de manifestations de masse $^{33}$, alors que les tensions interethniques sur le plan inter- 
individuel ne semblent pas se développer, même si, par ailleurs, les pratiques individuelles que l'on peut qualifier d'interethniques demeurent limitées.

La Constitution de 1974 assurait l'égalité entre les deux catégories de citoyens que recouvrait la distinction nation / nationalité ; de même elle affirmait la prééminence de la citoyenneté yougoslave sur la citoyenneté républicaine, ce qui constituait une garantie importante pour les minorités face à la "nation" majoritaire de telle ou telle République. On aurait donc pu s'attendre à une identification relativement importante à la catégorie "yougoslave" lors des recensements de la population. Ce ne sera le cas en 1981 que pour 5,6 \% de la population, pourcentage cependant le plus élevé depuis 1961, lorsque la création de la catégorie "Musulman" permet à ceux-ci de ne plus être classés dans cette catégorie (ou dans d'autres ethnies, voir supra). Ce pourcentage tombera à 3,3\% en 1991 lors du dernier recensement de la Yougoslavie fédérale, et sans doute cette baisse traduit-elle le repli identitaire sur sa communauté d'origine des années $80^{34}$. Le choix de l'appartenance à la "nationalité" yougoslave découlait d'un choix politique trans-ethnique émanant soit de certains membres des élites, soit et surtout, des familles mixtes par l'origine ethnique des parents. Rappelons cependant que cette identification n'était pas encouragée, notamment par l'absence d'espace politique où elle aurait pu s'exprimer, et qu'elle constituait même plutôt un handicap dès lors que l'on envisageait de mener une carrière de haut niveau.

Les mariages mixtes sont eux-mêmes relativement peu nombreux puisqu'en 198187 \% des familles étaient constituées de parents de "nationalité" identique dont $4 \%$ concernaient des familles où les deux parents se proclamaient yougoslaves, ce qui peut recouvrir en partie des couples mixtes. Les taux d'endogamie étaient ainsi les plus élevés (de 97 à 95 \%) parmi les Albanais, les Musulmans et les Slovènes, les plus faibles (de 84 à 73 \%) parmi les Hongrois, les Yougoslaves et les Monténégrins.

La faiblesse de l'identification à la "nationalité" yougoslave, ou celle des mariages mixtes, ne signifie pas pour autant la force des clivages ethniques. De multiples éléments montraient qu'il n'existait pas de discrimination d'origine ethnique dans la vie quotidienne. Les migrations inter-Républiques étaient loin d'être négligeables et ne se réduisaient pas au "retour" de membres d'une minorité d'une République vers la République où leur "nation" était majoritaire ; elles étaient avant tout déterminées par des motivations économiques ou culturelles (études) et l'installation dans une région autre que celle où sa nationalité était majoritaire n'avait rien d'anormal. De nombreuses enquêtes montrent que le plus souvent prévalait une distance interethnique limitée et que le sentiment de mauvaises relations interethniques était peu répandu ${ }^{35}$. Selon ces mêmes enquêtes menées au cours des années 80, les dissensions "nationales" ne constituaient pas la préoccupation première de la grande majorité de la population ; les principaux clivages ressentis étaient sociaux, entre une élite politico-administrative et la masse des autres, travailleurs et citoyens ${ }^{36}$. Néanmoins, d'autres enquêtes montrent l'importance, dès le milieu des années 80 et de façon croissante au cours des années ultérieures, de l'appartenance «nationale » dans les réponses à des questions spécifiquement politiques. La défiance croissante à l'égard des autres nationalités, le repli identitaire sur sa communauté-ethnie d'origine déboucheront alors sur des aspirations, pour les uns, à l'indépendance nationale ( «sortir au plus vite de cette Yougoslavie malade »), pour les autres, à un renforcement du rôle de l'État, notamment à son niveau fédéral, pour mettre un terme à ce processus de dislocation ${ }^{37}$. 
Mieux, si l'on peut dire, les élections pluralistes, qui se dérouleront séparément dans les différentes Républiques tout au long de l'année 1990, se traduiront par une adhésion massive des électeurs aux partis se présentant comme les champions des intérêts de la République concernée et de son ethnie majoritaire, ou, parallèlement, au(x) partis se posant comme le(s) défenseur(s) de la (les) minorité(s). Cette position partisane sur des bases ethniques atteindra la caricature en Bosnie-Herzégovine où chaque communauté ethnique votera, à une très large majorité, en faveur du parti se présentant comme son meilleur défenseur ${ }^{38}$. De même, lorsque les référendums concernant l'indépendance mettront en jeu des intérêts ressentis différemment, de façon même conflictuelle, par les différentes ethnies, comme en Croatie en 1991 et en Bosnie-Herzégovine en 1992, les choix partisans seront fonction de l'appartenance "nationale", allant d'un oui massif (supérieur à $90 \%$ ) à un refus de voter pour la minorité, le vote négatif étant presque inexistant.

Ces choix partisans ont ainsi nourri la transformation politique des tensions sociales en dissensions puis en hostilités interethniques (sans parler de leur prolongement militaire) alors même que les rapports interethniques individuels demeuraient globalement non conflictuels. Ce paradoxe ${ }^{39}$ montre combien la conscience citoyenne, en particulier yougoslave, était faible, combien elle fût occultée par l'identification à sa "nation", ultime refuge dans le contexte d'insécurité croissante sur le plan économique, social, politique.

Ce paradoxe renvoie donc à la faiblesse de l'espace politique permettant le développement d'une citoyenneté yougoslave: dans le cadre du régime communiste, monolithisme du Parti communiste, absence d'élection directe des principaux organes fédéraux, prédominance du principe paritaire et de la clef ethnique pour la répartition des principaux postes ; avec le pluripartisme de 1990, outre ceux des aspects antérieurs qui persistaient, quasi-absence de parti transnational, élections non organisées simultanément dans les différentes Républiques, absence de consultation populaire à l'échelle fédérale pour les questions engageant le devenir de la Fédération. Face à cette citoyenneté embryonnaire, les élites dirigeantes, après des années de lutte interne, s'emploieront, pour asseoir leur pouvoir régional, à paralyser le faible pouvoir fédéral qui persistait et à saper la confiance mutuelle entre les citoyens yougoslaves; ils y parviendront, ne serait-ce qu'en raison de leur légitimité découlant de leur contrôle des ressources économiques et sociales essentielles pour la vie quotidienne. De médiatique et politique, la "guerre civile" débouchera alors sur la guerre.

\section{Question nationale et citoyenneté non résolues}

51 La Yougoslavie fédérale n'est plus. Nombre de ses ressortissants se sont ainsi retrouvés orphelins, sans avoir clairement eu conscience auparavant de l'aboutissement vraisemblable des processus de désintégration, ni même et peut-être surtout, de quoi ils sont devenus orphelins faute d'avoir pu / voulu penser de quoi ils étaient partie prenante.

52 La dislocation de la Yougoslavie peut être vue comme la conséquence de l'absence de "peuple yougoslave" et de la persistance des clivages ethnico-culturels, voire confessionnels, entre les différents peuples; la Yougoslavie fédérale, plus précisément le monolithisme du Parti communiste, n'aurait que gelé la question nationale qui demeurait entière $\mathrm{e}^{40}$. Mais on peut aussi considérer que la désintégration de la 
Yougoslavie résulte de la façon dont a été utilisée la question nationale par le Parti communiste pour maintenir son pouvoir, en particulier avec la Constitution de 1974; l'institutionnalisation des intérêts locaux, censés pouvoir se contrôler mutuellement en raison de leur émiettement, a conduit, au bout d'une certaine période de stabilité, à l'aggravation des difficultés économiques et des tensions sociales auxquels le pouvoir central affaibli ne pouvait faire face ${ }^{41}$. Face au traitement "national" de ces tensions, la conscience citoyenne est apparue bien trop faible, faiblesse elle-même liée au monolithisme politique antérieur.

Dans cette perspective, les interrogations demeurent sur l'organisation politique souhaitable pour répondre à la question nationale et à l'émergence d'une citoyenneté non réduite à l'identité nationale. Elles le demeurent d'autant plus que les nouveaux États issus de la dislocation de la Yougoslavie comportent tous, Slovénie mise à part, des minorités importantes. C'est par l'établissement de réponses, plus ou moins explicites, à cette question que passera le retour à la paix et que sera déterminée en bonne part l'évolution vers la démocratie revendiquée par les dirigeants de ces nouveaux États.

\section{NOTES}

1. Witte (Bruno de), «Minorités nationales : reconnaissance et protection », Pouvoirs, (57), avril 1991. Cette position, largement répandue avant la montée des revendications nationales, n'est évidemment pas partagée par ceux qui ne voient dans le modèle yougoslave qu'une copie du modèle soviétique de "prison des peuples", comme M. Gjidara (Gjidara (Marc), "La solution fédérale: bilan critique », Pouvoirs, (57), avril 1991); précisons que ce dernier, universitaire français, est d'origine croate.

2. Voir notamment Rajakovic (Natacha), «Les ambiguités du yougoslavisme », in Rupnik (Jacques), éd., De Sarajevo à Sarajevo, l'échec yougoslave, Bruxelles: Complexe, 1992 et Krulic (Joseph), Histoire de la Yougoslavie, de 1945 à nos jours, Bruxelles : Complexe, 1993.

3. Cette transformation de facto de la royauté en Fédération ne prenait en compte au demeurant que les intérêts des Serbes et des Croates.

4. In V. Novak, 1948, cité par Batakovic (Dusan T.), « Le génocide dans l'État indépendant croate (1941-1945) », Hérodote, (67), octobre-décembre 1992.

5. Brubaker (Rogers), «L'éclatement des peuples à la chute des Empires, approche historique et comparative ", Actes de la Recherche en Sciences sociales, (98), juin 1993. Dans les Balkans, la question de l'homogénéité ethnique des territoires des nouveaux États-Nations se posait au regard de l'organisation administrative de l'Empire ottoman qui regroupait des communautés de peuple et de religion en millets auxquels n'était pas attribué un territoire défini, Hottinger (Arnold), « Milliyet ottoman et nation européenne dans les Balkans », Liber supplément à Actes de la Recherche en Sciences sociales (105), décembre 1994.

6. Pavlowitch (Stevan K.), "L'héritage titiste", in Rupnik (Jacques), éd., op. cit. Ainsi, le maintien de l'état d'urgence au Kosovo jusqu'en 1966 n'a guère permis à la question albanaise d'apparaître au grand jour avant les premières manifestations politiques de 1968 (cf. Roux (Michel), Les Albanais en Yougoslavie. Minorité nationale, territoire et développement, Paris : Maison des Sciences de l'Homme, 1992). 
7. Cette position initiale trans-nationale, conforme à l'idéologie communiste, n'excluait pas, bien entendu, une participation à cette résistance variable selon les nationalités.

8. La distinction n'était pas, le plus souvent, juridiquement établie. Mais la Constitution croate en vigueur jusqu'en 1990 précisait que la Croatie était « l'État des Croates et des Serbes ainsi que des minorités ", affirmant ainsi son caractère multinational ; cette reconnaissance juridique des Serbes en Croatie, spécifiée à cause de la politique oustachie de 1941, sera supprimée dans la nouvelle Constitution croate de 1990, ce qui sera ressenti par les Serbes comme une agression.

9. D'où son appellation fréquente ces dernières années de "mini-Yougoslavie".

10. Sur cette distinction concernant les minorités voir Kende (Pierre), "Comment gérer le problème des minorités en Europe centrale et orientale", Notes et études documentaires, (4964-4965), 1992. "Nation"-narod et "nationalité"-narodnost correspondent donc à une réalité ethnico-culturelle et non pas, contrairement à la pratique française, à une réalité politique, celle-ci correspondant à la citoyenneté nommée državljanstvo, en référence à l'État (država).

11. Outre les six Républiques, la Fédération se composait de deux Provinces autonomes, Kosovo et Voïvodine, toutes deux faisant partie de la République de Serbie.

12. Pour ne pas alourdir les propos, nous ne retenons comme cadre juridique que la Constitution fédérale de 1974, notamment ses articles 170-171 et 244 à 250 , en nous bornant à signaler les évolutions significatives depuis la Constitution de 1946.

13. Malgré les affirmations qui se sont développées dans le récent contexte nationaliste tendant à légitimer les nouveaux États, notamment par l'existence d'une langue "nationale" spécifique, Serbes et Croates parlent la même langue (origine et grammaire identique, compréhension sans ambiguïté en l'absence de traducteur etc.) même s'il existe un alphabet différent et des différences mineures dans le vocabulaire et la syntaxe. A fortiori en va-t-il de même pour les Musulmans; quant à l'émergence prétendue d'une langue "bosniaque", elle renvoie au mieux à un parler régional du serbo-croate.

14. Pour la constitution en ethnie puis en "nation" des musulmans "d'origine yougoslave", c'est-à-dire ceux ne relevant pas de minorités, par ailleurs reconnues (albanaise, turque, tsigane pour l'essentiel), les prescriptions des recensements sont éclairantes. En 1948, ils pouvaient se déterminer, à partir de leur identité religieuse, comme Serbes-musulmans, Croates-musulmans ou musulmans indéterminés; dans les deux premier cas, ils étaient assimilés aux Serbes ou aux Croates. En 1953, ceux qui se proclamaient musulmans étaient regroupés dans la catégorie "Yougoslaves indéterminés". En 1961 apparaît la nouvelle catégorie de "Musulman", au sens ethnique et non religieux comme le précise le recensement; cette nouvelle ethnie sera transformée en "nation" avec le recensement de 1971. Malgré cette évolution, le principe identitaire mis en avant est culturel, d'origine religieuse, puisque seule la religion permet, au moins à l'origine, de distinguer cette communauté ; ceci correspondait cependant, à l'époque ottomane, à une différenciation socio-économique et politique, la population s'étant islamisée l'ayant fait avant tout pour des avantages socio-économiques. Sur les musulmans en Yougoslavie, voir Popovic (Alexandre), Les Musulmans yougoslaves, 1945-1989, médiateurs et métaphores, Lausanne: L'âge d'homme, 1990 ; Popovic (Alexandre), "Problèmes d'approche de l'Islam yougoslave ", in Les musulmans des Balkans à l'époque post-ottomane, Istanbul : Isis, 1994 ; ainsi que Bougarel (Xavier), «Bosnie-Herzégovine : anatomie d'une poudrière », Hérodote, (67), octobredécembre 1992.

15. Nikolic (Pavle), «L'institution du chef de l'État en Serbie et en Yougoslavie ", Revue d'études comparatives Est-Ouest, 23 (4), décembre 1992 et Goati (Vladimir), «La démocratie majoritaire dans l'espace yougoslave ", Peuples méditerranéens, (61), octobre-décembre 1992. Certains (comme Katunaric (Vjeran), « Multi-ethnic Yugoslavia and Political Change with Particular Refenrence to Croatia », Peuples méditerranéens, (61), octobre-décembre 1992) parlent de semi-Confédération, une telle dénomination montrant bien les ambiguïtés des institutions. 
16. Cette ambiguïté sera au centre de débats juridico-politiques en 1991 sur le droit à la sécession et l'intangibilité des frontières de chaque République. Les partisans du droit à la sécession "oublieront" ainsi les limites posées par cet alinéa 3 de l'article 5 ; voir par ex. Gjidara (Marc), «La Croatie en 1992. Une affirmation difficile », Notes et études documentaires, (4984), 1993.

17. Nous n'abordons pas ici le gouvernement fédéral dont le rôle, conformément au modèle soviétique, était presqu'exclusivement technique; il ne jouera un rôle politique notable qu'à partir de 1989, dans le contexte de l'effondrement du Parti communiste et de la paralysie de la Présidence.

18. L'épisode de la nomination de S. Mesić, représentant de la Croatie, illustre de façon caricaturale cette paralysie. Devant devenir selon le principe de rotation le président du collège présidentiel en mai 1991, son élection sera dans un premier temps bloquée par les représentants serbes et monténégrins ; ceux-ci disposaient en effet de la moitié des voix (4 sur 8) depuis que la Serbie avait repris le contrôle de ses deux provinces autonomes en 1989 et que le Parti communiste n'y était plus représenté compte tenu de sa dislocation en 1990. Leur opposition à la nomination de S. Mesić découlait de l'appartenance de ce dernier à la HDZ, parti nationaliste ayant gagné les élections de 1990 en Croatie et prônant l'indépendance. Lui-même ayant déclaré qu'il serait le dernier Président de la Fédération, il sera nommé Président sur pression de la CEE et commentera sa démission en décembre 1991 par: "J'ai rempli mon devoir, la Yougoslavie n'existe plus ».

19. Parti dit «réformateur » du Premier ministre fédéral, A. Marković, croate se proclamant yougoslave, qui n'obtiendra quelque résultat significatif qu'en Bosnie-Herzégovine. Le projet de faire élire les représentants du Conseil fédéral sur une base fédérale s'inscrit plus largement dans les débats des années 1990-1991 concernant une réforme constitutionnelle autour de l'alternative Fédération-Confédération; l'option fédérale était avancée notamment par des Serbes, disposant d'une majorité démographique relative ( $40 \%$ de la population de l'ex-Yougoslavie) mais rejetée par les Croates $(20 \%)$ et les Slovènes $(8 \%)$, partisans d'une Confédération, quelque peu irréelle au vu du caractère confédéral déjà existant, voir Crnobrnja (Mihailo), Le drame yougoslave, Rennes : Apogée, 1992. L'option fédérale serbe ne découle pas seulement de leur importance démographique; ils avaient un intérêt existentiel à la sauvegarde de la Yougoslavie et au renforcement du pouvoir central en raison de leur dissémination hors de la Serbie, Bolcic (Silvano), "La sociologie et la "guerre intérieure" ", Peuples méditerranéens, (61), octobredécembre 1992.

20. Pavlowitch (Stevan K.), art.cit.

21. Rappelons, à titre de comparaison, que depuis l'Acte unique de 1985, la plupart des décisions du Conseil de l'Union européenne sont prises selon la règle de la majorité dite "qualifiée", l'unanimité n'étant requise que pour des domaines importants précisément définis. Par ailleurs, le Parlement européen découle d'une représentation pondérée des différents États membres.

22. Krulic (Joseph), op.cit. Ainsi le tournant paraît être le "plan de stabilisation" de 1983, date à partir de laquelle le produit social sera globalement stagnant alors qu'il progressait depuis le milieu des années 1950 à un rythme supérieur à $6 \%$ l'an ; le salaire réel moyen baissera de $30 \%$ de 1978 à 1988, retrouvant alors son niveau de 1968, voir Statistički Godišnjak, 1990.

23. Il existait en effet un déséquilibre démographique, territorial et historique en faveur de la Serbie. Notamment, cette dernière a été, avec le Monténégro, un État indépendant reconnu dès 1878 et a fait partie des vainqueurs de la Première Guerre mondiale ; sans doute peut-on y voir une source essentielle de ce déséquilibre par rapport à d'autres peuples de la Yougoslavie, comme les Slovènes et les Croates, pour lesquels demeurait non résolue la question de leur émancipation / indépendance.

24. Schnapper (Dominique), La communauté des citoyens, Paris : Gallimard, 1994. 
25. Popovic (Alexandre), articles cités ; voir notamment "Récits de pèlerinage à la Mecque » (1994) où l'auteur montre par des extraits de la presse musulmane toutes les ambiguïtés découlant de la distinction musulmans/Musulmans.

26. A. Izetbegović, comme d'autres membres fondateurs du SDA, fut membre dans les années 1940 d'un groupe panislamiste Mladi Muslimani ; sur le développement d'un courant panislamiste en Bosnie-Herzégovine et son influence déterminante à partir de la fin des années 1980, voir Bougarel (Xavier), «Un courant panislamiste en Bosnie-Herzégovine », in Kepel (Gilles), éd., Exils et royaumes. Les appartenances au monde arabo-musulman aujourd'hui, Paris : PFNSP, 1994.

27. Sur cette crise, voir Krulic (Joseph), op.cit.

28. Avec ambiguïté de l'expression découlant du double statut de Belgrade, capitale de la Serbie et capitale fédérale ; c'est évidemment ce dernier aspect qui en l'occurrence était déterminant, mais l'expression par son ambiguïté permettait d'alimenter le discours nationaliste en lui donnant une dimension interethnique implicite.

29. Malgré l'objectif de convertibilité du dinar retenu dans le cadre de la Réforme de 1965, l'allocation des devises nécessaires aux importations continuera à se faire pour une large part de façon administrative, selon des règles établies bien entendu à l'échelle fédérale et reflétant avant tout la pénurie de devises. Mais la Croatie s'estimait "exploitée" par la répartition prévalant jusqu'alors, considérant que les rentrées de devises dues au tourisme (qu'elle concentrait puisqu'elle comprend la quasi-totalité de la côte dalmate) et aux envois des travailleurs émigrés originaires de cette République devaient lui être attribuées dans une plus forte proportion, voire en totalité. Bon exemple de discours nationaliste à rhétorique économique qui resurgira de façon récurrente jusqu'en 1990, y compris de la part des Serbes et des Slovènes.

30. Avec notamment le projet d'une nouvelle Constitution de Croatie comportant une déclaration de souveraineté, d'une monnaie propre et du contrôle par la Croatie de tous ses revenus, sa contribution à une éventuelle Fédération reposant sur une base volontaire.

31. Katunaric (Vjeran), "Jugoslavija kao etničko društvo » (La Yougoslavie en tant que société ethnique), Sociologija, 24 (2-3), 1982. Au sommet de la Fédération, ce principe d'équilibre nationaléthnique souffrira de quelques exceptions ; ainsi, tous les chefs de gouvernement seront croates à partir de 1974 alors que les ministres de l'Intérieur seront serbes, Krulic (Joseph), op.cit. Il faudra attendre en 1989 la nomination d'A. Marković, lui-même croate, à la tête du gouvernement pour que celui-ci ne soit plus composé d'abord en fonction de cette règle ethnique.

32. Pour reprendre l'expression de Pavlowitch (Stevan K.), art.cit.

33. On pense ici aux manifestations de masse en Serbie et au Monténégro à partir de 1987 qui culmineront en 1989 avec la célébration du sixième centenaire de la bataille (défaite) du Kosovo, l'un des mythes fondateurs de la nation serbe; en faire l'origine du renouveau nationaliste qui conduira à la dislocation de la Yougoslavie, comme on peut le trouver fréquemment sous des plumes occidentales ou dans les propos de Slovènes et de Croates, revient cependant à négliger le nationalisme, moins spectaculaire mais non moins vif, des autres "nations" et de leurs élites, notamment avec leur refus d'envisager une modification des relations constitutionnelles entre la Serbie et ses Provinces autonomes, à l'origine du renouveau du nationalisme serbe.

34. Tous les éléments statistiques sont tirés de Savezni Zavod za Statistiku: Statistički Godišnjak Jugoslavije, 1990 et Statistički bilten, (1934), 1992.

35. Voir Bolcic (Silvano), art.cit.; Katunaric (Vjeran), art.cit., est amené aux mêmes conclusions à partir d'enquêtes faites en Croatie en 1985 et 1989, sans noter de changements sensibles entre ces deux dates. Voir également pour la Bosnie-Herzégovine Bougarel (Xavier), «Bosnie-Herzégovine... », art.cit.

36. Bolcic (Silvano), art.cit. On peut néanmoins s'interroger sur la portée de ces enquêtes, qui peuvent refléter, d'abord, des décennies de discours officiel pluriethnique dans un contexte de liberté d'expression à tout le moins surveillée; de même, la représentation de la stratification 
sociale semble correspondre avant tout au caractère monolithique du pouvoir politique prévalant jusqu'en 1990.

37. Dans cette prédominance du traitement ethnique des conflits, la ligne de partage s'est très vite dessinée entre les Serbes et les Monténégrins d'une part, les autres d'autre part.

38. Le mode de scrutin majoritaire, retenu en Serbie, Croatie et Macédoine a lui-même accentué la coupure entre majorité et minorité et donc entre ethnies; la combinaison du mode majoritaire-proportionnel en Bosnie-Herzégovine s'est traduit par la sur-représentation au Parlement des partis nationalistes au détriment des partis transethniques, ceux-ci n'ayant obtenu, au total, que $20 \%$ environ des voix, Goati (Vladimir), art.cit.

39. Paradoxe expliquant que rares ont été ceux qui avant 1990 ou même 1991 ont perçu la possibilité que le processus de dislocation aille à son terme, a fortiori qu'il débouche sur une guerre civile. Il permet également de comprendre que face à cette évolution prédominent le désarroi des sociologues (voir notamment les contributions de Bolcic (Silvano), art.cit. et Katunaric (Vjeran), art.cit. dans Peuples Méditerranéens) comme le sentiment d'impuissance fataliste pour une très grande majorité de la population, même si ses choix politiques partisans ont le plus souvent nourri ce processus.

40. Position partagée par exemple par M. Gjidara (Gjidara (Marc), art.cit., 1991), qui réduit l'expérience yougoslave à une variante du modèle soviétique et par V. Goati (Goati (Vladimir), art.cit.), pour qui la dislocation de la Yougoslavie renvoie aux pratiques des élites des Républiques / Provinces dans le cadre de la décomposition du régime sociopolitique de la période communiste.

41. Voir par exemple Pavlowitch (Stevan K.), art.cit. et Bougarel (Xavier), art.cit., 1992.

\section{RÉSUMÉS}

Dans la Yougoslavie fédérale, chaque république disposait de sa nation, hormis la BosnieHerzégovine. L'existence de minorités nationales implique deux catégories de citoyens, bien que l'égalité des citoyens ait été proclamée et que la nation ne soit pas liée à un territoire. Les structures institutionnelles et les modes de décision font que la Yougoslavie ressemblait davantage à une confédération. Les crises nationales ont été résolues par la répression et la la satisfaction des demandes formulées. L'évolution confédérale du système politique yougoslave conduira à une interprétation nationale des divergences économiques et des conflits sociaux. La dislocation de la Yougoslavie résulterait-elle donc de la manière dont a été utilisée la question nationale par le parti communiste pour se maintenir au pouvoir?

In federal Yugoslavia, each Republic owned its nation, but Bosnia and Herzegovina. Distinct categories of nation and national minorities involve two sets of citizens, although the equality of citizens was claimed and nation was not territorially defined. Because of institutional structures and decision making, Yugoslavia looked more like a confederation rather than a federation. National crises were repressed, but their aims were, more or less, fulfilled. Confederal evolution of the Yugoslavian political system will lead to an ethnic explanation of the economic inequality and social conflicts. Is the demise of Yugsolavia a consequence of the way by which the national question was used by the communist party to remain on power? 
INDEX

Index géographique : Yougoslavie

Mots-clés : Citoyenneté, Question natioanle, Fédération, Confédération

\section{AUTEUR}

\section{MICHEL DROUET}

M. Drouet est professeur d'économie à l'Université de Rennes 2. 